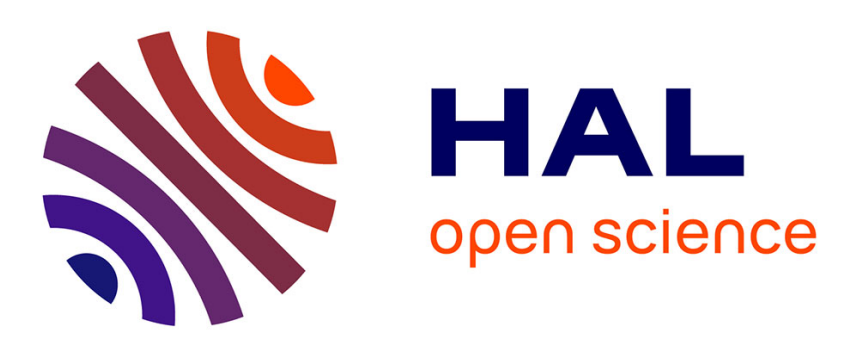

\title{
Le français calédonien (Nouvelle-Calédonie). Description d'un français régional, confrontations de terrains panfrancophones
}

Christine Pauleau

\section{- To cite this version: \\ Christine Pauleau. Le français calédonien (Nouvelle-Calédonie). Description d'un français régional, confrontations de terrains panfrancophones. Langages, 2016, 203, pp.5-20. 10.3917/lang.203.0005 . hal-01987738}

\author{
HAL Id: hal-01987738 \\ https://hal.parisnanterre.fr/hal-01987738
}

Submitted on 21 Jan 2019

HAL is a multi-disciplinary open access archive for the deposit and dissemination of scientific research documents, whether they are published or not. The documents may come from teaching and research institutions in France or abroad, or from public or private research centers.
L'archive ouverte pluridisciplinaire HAL, est destinée au dépôt et à la diffusion de documents scientifiques de niveau recherche, publiés ou non, émanant des établissements d'enseignement et de recherche français ou étrangers, des laboratoires publics ou privés. 


\section{Le français calédonien (Nouvelle-Calédonie). Description d'un français régional, confrontations de terrains panfrancophones}

\section{SITUATION DU FRANÇAIS EN NOUVELLE-CALÉDONIE ${ }^{1}$}

La Nouvelle-Calédonie est une collectivité française inscrite de fait dans la francophonie, mais dont la langue française suscite peu d'intérêt, en comparaison notamment des formes de français parlées ailleurs dans le monde, sur le continent américain ou africain par exemple. Sa situation géolinguistique et sociolinguistique étant peu connue, nous commencerons par en dessiner les grandes lignes.

\subsection{Situation géo-linguistique}

L'archipel de la Nouvelle-Calédonie, le plus lointain territoire ultramarin français ${ }^{2}$, est, depuis 1999, une collectivité d'outre-mer (ex-territoire d'outre-mer ${ }^{3}$ ). Situé dans l'océan Pacifique sud, entre le tropique du Capricorne et l'équateur, il faut pour y accéder depuis la France deux mois de navigation, en moyenne vingt-cinq heures de voyage par avion. $17000 \mathrm{~km}$ et dix heures de décalage

\footnotetext{
1. Nous tenons à remercier ici les relecteurs anonymes de la revue Langages pour leurs remarques particulièrement constructives.

2. Distance en km des territoires ultramarins à Paris, selon l'IRD (2012:15) : Guadeloupe : 6 761, NouvelleCalédonie : 16 758, Polynésie : 15 727, Réunion : 9342.

3. «[...] la Nouvelle-Calédonie échappe au statut général des collectivités locales défini par le titre XII de la Constitution. Toutefois, la révision constitutionnelle de 2003 l'intègre à la liste des collectivités d'outre mer (article 72-3). [...] la Nouvelle-Calédonie est une collectivité « sui generis ». Dans ce cadre, on emploie l'expression « collectivité d'outre mer à statut particulier » pour la désigner. » (www.collectivites-locales.gouv. fr/statuts-nouvelle-caledonie-et-polynesie). Voir les annexes pour plus de détails.
} 
horaire séparent Nouméa de Paris. En termes de géographie humaine, le territoire calédonien fait partie de la Mélanésie ${ }^{4}$ et comptait en 2009245580 habitants ${ }^{5}$. L'économie s'appuie surtout sur l'extraction et la production du minerai de nickel ${ }^{6}$, ainsi que sur le secteur tertiaire ${ }^{7}$. La croissance soutenue et stable depuis les années 90 entraîne un taux d'emploi qui est le plus fort de l'outre-mer français ${ }^{8}$.

Ce pays francophone océanien de moins de $19000 \mathrm{~km}^{2}$, soit un peu plus de deux fois la Corse, est le voisin immédiat de territoires anglophones ${ }^{9}$ imposants tels que l'Australie $\left(7693000 \mathrm{~km}^{2}\right)$, qui se trouve à $1800 \mathrm{~km}$ de la NouvelleCalédonie, et la Nouvelle-Zélande $(267000$ km²), à $1700 \mathrm{~km}$. Tahiti, terre francophone, se trouve beaucoup plus loin, à $5000 \mathrm{~km}$. La méconnaissance dont l'archipel calédonien est l'objet est-elle due à ce caractère d'îlot lointain, moins familier des Français que le Maghreb ou l'Afrique, eux, bien connus du fait de leur proximité géographique, leur présence dans l'Hexagone par l'immigration, le nombre des locuteurs concernés ? Ces trois paramètres jouent exactement à l'inverse pour la Nouvelle-Calédonie, qui se trouve aux antipodes, ne connaît que peu de migration vers la France et concerne moins de 300000 locuteurs. Pourtant la francophonie calédonienne se distingue par d'autres aspects, au moins aussi intéressants a priori, notamment par sa proportion de " francophones réels ${ }^{10}$ », laquelle est particulièrement forte par opposition aux « francophones partiels » : 654000 francophones océaniens dénombrés par l'OIF (2007), avec une proportion de $90 \%$ de francophones réels due à un taux de scolarisation très élevé (OIF 2005), ce qui constitue une originalité rapprochant la Nouvelle-Calédonie des pays de la francophonie dite « du nord » alors qu'elle a, du fait de son passé colonial, un statut de pays de francophonie «du sud ${ }^{11}$. Ainsi la francophonie dans ces régions n'est-elle pas un fantasme, le français y devenant, en zone

4. Voir la carte des aires culturelles en annexe.

5. Données ISEE (2011).

6. La Nouvelle-Calédonie se place au troisième rang mondial des réserves estimées.

7. $80 \%$ environ de la création de richesse - chiffres de 2009 (IRD, 2012 : 149).

8. La croissance économique résiste encore à la crise mondiale du fait de la récente construction de deux usines métallurgiques et le fort taux d'emploi (57,9\%) reste réel malgré les vicissitudes d'un marché du nickel capricieux, explosant par exemple après 2002 mais déclinant fortement en 2009 (IRD, 2012 : 149).

9. La colonisation ayant été massivement anglaise en Océanie.

10. Les études dans ce domaine comptent en moyenne au moins deux ans de scolarisation pour un francophone dit « potentiel» (ou « partiel») et au moins six ans pour un francophone dit « réel » (Queffélec 2000). Selon l'Organisation Internationale de la Francophonie - OIF - (2011), on distingue « francophones » («personnes capables de faire face, en français, aux situations de communication courante ») et « francophones partiels » (« personnes ayant une compétence réduite en français, leur permettant de faire face à un nombre limité de situations »).

11. Bien que d'autres groupements existent, il est courant en sociolinguistique de la francophonie de classer les régions francophones en deux groupes, ceux de la francophonie « du nord », comprenant les régions du berceau de la langue française comme la France et la Belgique ainsi que celles dans lesquelles le français est d'implantation ancienne comme le Québec, et ceux de la francophonie « du sud », désignant les régions dans lesquelles la langue française a été importée plus récemment par la colonisation. 
urbaine, la langue vernaculaire des locuteurs nés de parents non francophones de langue maternelle (nous y reviendrons infra).

Le point de vue géo-linguistique ne livre apparemment pas de réponse immédiate convaincante pour expliquer le manque d'intérêt à l'égard de cette partie de la francophonie, mais d'autres facteurs peuvent intervenir, en particulier du côté de son histoire.

\subsection{Situation historique et sociolinguistique}

Les Kanak ${ }^{12}$, peuple noir mélanésien, arrivent dans l'archipel il y a environ 3500 ans. Les vingt-huit langues kanak de Nouvelle-Calédonie relèvent, parmi les langues océaniennes, de la famille austronésienne orientale ${ }^{13}$. À partir de la fin du XVIII ${ }^{\mathrm{e}}$ siècle, elles sont mises en contact avec d'autres langues (on en compte plus d'une vingtaine en deux siècles) telles que l'anglais, les langues asiatiques, les langues polynésiennes et le français à partir du milieu du XIX $X^{\mathrm{e}}$ siècle. Ce sont les navigateurs ${ }^{14}$, les commerçants australiens et asiatiques, les missionnaires polynésiens et anglais de la London Missionary Society, qui établissent les premiers contacts avec la population autochtone mélanésienne (les premières familles métisses calédoniennes sont souvent issues d'unions entre marins anglo-saxons et femmes kanak). La langue de communication entre occidentaux et « indigènes » est alors un pidgin anglo-mélanésien qui mélange l'anglais, les langues polynésiennes et les langues kanak : le bichelamar. Jusqu'à la fin $\mathrm{du}$ XIX ${ }^{\mathrm{e}}$ siècle, cet idiome reste en concurrence avec le français et l'anglais en Nouvelle-Calédonie (le bichelamar est aujourd'hui l'une des langues officielles de l'archipel voisin, le Vanuatu).

Avant 1853, les premiers immigrants sont surtout anglo-saxons, comme le santalier James Paddon, qui donne son nom aux colons australiens de cette période, les colons Paddon, une dizaine de familles dont certaines sont d'origine allemande. Entre 1850 et 1925, un certain nombre de francophones débarque à Tahiti avant, pour certains, de se diriger vers la Nouvelle-Calédonie (Corne, 1979 : 632), originaires principalement « du Grand Centre et de l'Ouest de la France » (54\%) ainsi que de Bretagne et d'Anjou (22,5\%), du Poitou et de Saintonge (10\%) (voir Hollyman, $1979: 633){ }^{15}$. La « prise de possession » par la

12. Le mot Kanak (n.pr. ou adj.) est employé ici dans le sens de 'Mélanésien'. Même si ce mot a encore parfois une valeur politique dans certaines situations, il est d'un usage de plus en plus courant sans valeur politique particulière.

13. Les langues mélanésiennes kanak de Nouvelle-Calédonie sont des langues à tradition orale. En voici la liste alphabétique : ajië, arhâ, arhô, bwatoo, caac, cèmuhî, dehu, drubea, faga uvea, fwâi, iaai, jawe, kumak, neku, nélêmwa, nemi, nénéma, nengone, nerë, numèè, nyelâyu, orowe, paicî, pije, pwaamei, pwapwâ, sîchë, tîrî, xârâcùù, yuaga.

14. L'Écossais James Cook est le premier occidental à découvrir en 1774 l'île des Pins, au sud de l'archipel. Il donne à ce dernier le nom de Nouvelle-Calédonie, dans le sens de «nouvelle Écosse ».

15. Hollyman tire ces chiffres de l'analyse d'un dépouillement portant sur 600 immigrants. 
France en 1853 confirme l'entrée de la langue française dans le paysage linguistique calédonien, qui se modifie alors en fonction de nombreux changements démographiques. La colonisation française entraîne dans l'archipel, alors peuplé d'au moins 60000 Kanak $^{16}$, la venue d'immigrés de tous horizons. Une population pénale et une population libre se côtoient de 1863 à 1897 : la colonie pénitentiaire de la Nouvelle détient des transportés, criminels de droit commun (22 000), des déportés, prisonniers politiques comme les communards autour de Louise Michel (4 000) ${ }^{17}$. De tout l'Empire colonial arrivent des bagnards (30 000 en tout) qui, à partir de 1885, peuvent aussi être de simples délinquants récidivistes (les relégués). D’Algérie notamment sont envoyés quelque 2000 détenus qui feront souche en "Calédoune », nom qu'ils donnent à l'archipel calédonien. Le peuplement de la colonie est assuré par des unions entre anciens prisonniers et prisonnières (2 000 femmes sont envoyées à La Nouvelle) ou avec des femmes kanak. Du fait de l'obligation de résidence perpétuelle dans la colonie (dont se double en général la condamnation au bagne) et la réhabilitation des condamnés après leur peine prévue par la loi (qui leur accorde des concessions à cultiver), certains ex-détenus s'installent en tant qu'agriculteurs ${ }^{18}$. D'autres sont mineurs lorsque débute l'exploitation du nickel (1875), les premiers mineurs étant majoritairement des bagnards, côtoyant par la suite des travailleurs kanak, néo-hébridais, japonais, tonkinois, chinois, javanais ${ }^{19}$. « Le caractère bigarré de la population néo-calédonienne avec sa composante asiatique, découle [...] de l'activité minière » (Gay, $2014: 51)^{20}$. Le bagne est un autre facteur déterminant le peuplement, il est « à l'origine du groupe le plus nombreux de l'immigration rurale [...], les condamnés et leur famille constituent [au XIX ${ }^{\mathrm{e}}$ siècle] la majorité de la population européenne en brousse » (Ibid.), la population libre étant surtout à Nouméa. Cette dernière vient de France, mais aussi d'Australie, d'Océanie, d'Asie, d'Indonésie ou d'autres îles comme la Réunion (l'île Bourbon). La colonie de peuplement implantée (implantation qui succède à la colonie pénitentiaire fermée en 1897) subit une « crise profonde du projet de peuplement colonial » (IRD, 2012 : 110), à la fin du XIXe siècle. Malgré l'immigration planifiée de planteurs de l'île Bourbon (avec leurs coolies indiens dits malabar) et d'éleveurs d'Alsace-Lorraine, les pénaux sont toujours deux fois plus nombreux que les colons libres. La population kanak a dramatiquement chuté (27 000 en

16. Les chiffres officiels ayant probablement été minimisés (IRD, 2012 : 108).

17. Les chiffres, jusqu'à la fin de ce paragraphe, sont empruntés à IRD (2012: 107-110).

18. Cependant, moins du dixième des bagnards s'installe dans le pays (Gay, $2014: 51$ ).

19. Ces mineurs constituent un prolétariat qui ne peut se déplacer librement dans la colonie, surtout dans les centres urbains de brousse et à Nouméa, qu'il s'agisse des Kanak soumis au code de l'indigénat, des anciens bagnards ou des travailleurs engagés océaniens et asiatiques (Gay, 2014 : 55).

20. En 1936, cette population asiatique et javanaise venue pour la mine constitue toujours $13 \%$ de la population (IRD, $2012: 110)$. 
1901) ${ }^{21}$, celle des Européens peine à progresser (23 500). Les échecs agricoles et l'insuffisance de l'emploi minier font migrer les Européens vers l'Australie, les Nouvelle-Hébrides ou la France. Malgré les dernières tentatives de peuplement (avec l'envoi, dans les années 20, de 240 colons nordistes fuyant le nord de la France dévasté par la guerre et s'installant, pour certains, durablement) en 1945, les Européens ne sont plus que 18 500. Dans ce climat d'anémie démographique séjournent, entre 1942 et 1946, près d'un million de soldats américains, ainsi que les forces alliées australiennes et néo-zélandaises, marquant profondément la culture calédonienne. À cette époque, avec l'abolition du régime de l'indigénat et la résidence libre accordée aux engagés (1946), la société multiculturelle calédonienne est en pleine émancipation et le français commence à devenir la langue de communication du fait de la multiplication des échanges inter-groupes. Cela se poursuit par l'arrivée, dans les années 60, de colons (fonctionnaires, administrateurs, hommes d'affaires...) qui convergent des ex-colonies devenues indépendantes vers la Nouvelle-Calédonie, toujours française. Le « boum économique » (1969-1972) lié à l'exploitation du nickel accentue fortement cet accroissement des migrations, en provenance notamment de la métropole, des départements français d'outre-mer (Antilles, Martinique surtout, Guyane, Réunion...), de Polynésie française (Tahiti, et surtout Wallis et Futuna) ou d'anciens territoires français (Djibouti, Comores, Algérie, etc.).

Ainsi une mosaïque ethnolinguistique résulte-t-elle des migrations multiples au cours de l'histoire, les communautés les plus nombreuses étant aujourd'hui celle des Kanak, puis celle des Européens, puis celle des Wallisiens (Polynésiens de Wallis et Futuna). En effet, la répartition selon la communauté d'appartenance (ISEE 2011) compte :

- 40,3\% de personnes se déclarant Kanak

- 29,2\% se déclarant Européens

$-8,7 \%$ se déclarant Wallisiens-Futuniens

- $8,3 \%$ se déclarant Métis

- 7,3\% déclarant appartenir à d'autres communautés

- $5 \%$ se déclarant Néo-Calédoniens

- 1,2\% ne déclarant aucune communauté d'appartenance

Les Kanak et les Kanak métisses représentent $44,3 \%$ de la population, les Européens et les Européens métisses 33,9\%, les Wallisiens et Futuniens et les Wallisiens et Futuniens métisses $10,4 \%{ }^{22}$.

21. Le choc épidémiologique datant de la période des premiers contacts (XVIII ${ }^{\mathrm{e}}$ siècle), les pertes humaines conséquentes aux insurrections, la dépression collective sont notamment à l'origine de cette chute démographique.

22. En 1996, la part des Kanak était de 44,1\%, celle des Européens de 34,1 \% et celle des Wallisiens et Futuniens de 9,0\% (ITSEE \& INSEE 1997). Précisons toutefois que ces chiffres sont à prendre avec réserve étant donné les distorsions qui existent souvent entre la réalité des communautés et des métissages et les représentations de ceux-ci (qui notamment poussent les recensés métis à « choisir » de déclarer telle ethnie plutôt que telle autre pour le recensement). 
Le français, qui est la langue maternelle des Européens et d'un nombre croissant de membres d'autres groupes linguistiques, est aussi langue seconde pour les locuteurs de langue maternelle kanak, polynésienne (tahitien, wallisien, fidjien, etc.), indonésienne (javanais...), asiatique (vietnamien, mandarin, etc.), ainsi que pour les locuteurs du créole tayo et du bichelamar ${ }^{23}$. On ne peut cependant omettre dans ce paysage linguistique l'influence de l'anglais, le poids de l'anglais austral voisin (et celui de la présence américaine pendant la Seconde Guerre mondiale), étant aujourd'hui doublé par la domination anglophone mondialisante.

Certaines langues kanak sont actuellement intégrées dans l'enseignement primaire, secondaire et universitaire. Au lycée, notamment, l'enseignement du dehu, du nengone, du paîci, de l'ajië est officielle depuis 1992 et fait l'objet d'épreuves orales et écrites du baccalauréat ${ }^{24}$. Le français, seule langue officielle, est néanmoins la langue d'enseignement. Aujourd'hui, et de plus en plus depuis la deuxième moitié $\mathrm{du} X X^{\mathrm{e}}$ siècle, le français est aussi langue véhiculaire, i.e. langue de communication entre les divers groupes linguistiques : il sert des fonctions prestigieuses (langue de la parole publique, des situations formelles) et des fonctions ordinaires (langue de la parole privée, langue du quotidien) dans le groupe européen mais aussi, de plus en plus, dans les autres groupes et, notamment, dans les couples mixtes. En conséquence, au moins en milieu urbain, cet idiome est en train de se vernaculariser, de prendre la place des langues vernaculaires kanak, polynésiennes, indonésiennes, asiatiques : pour nombre de locuteurs issus de parents bilingues (par exemple, langue 1 : langue kanak / langue 2 : français ou encore langue 1 : langue polynésienne / langue 2 : français, etc. ${ }^{25}$ ), le français est, surtout depuis les années 90 , la langue courante du quotidien. Ces locuteurs citadins deviennent donc peu à peu francophones monolingues et leurs enfants peuvent avoir le français pour unique langue maternelle ${ }^{26}$ (notons qu'en 1996, 30 \% des Kanak sont déjà des citadins et, dans les autres ethnies, une grande majorité l'est aussi ; voir ITSEE \& INSEE 1997).

La situation du français en Nouvelle-Calédonie est d'autant plus originale qu'elle s'inscrit dans une dynamique post-coloniale unique au monde : au sein de ce pays tardivement colonisé, et surtout tardivement peuplé de francophones

23. Voir ici même l'article d'Ehrhart au sujet du tayo, d'une part, et des langues déclarées au cours des recensements, d'autre part, et de la prudence avec laquelle on devrait en déduire un « paysage linguistique ».

24. À l'Institut de Formation des Maîtres (IFM) et à l'école Normale du Privé (ENEP), deux Unités d'Enseignements concernent les langues et cultures autochtones. À l'Université de la Nouvelle-Calédonie (UNC) une Licence Langues et Cultures Régionales a été ouverte depuis 2001.

25. Situation ordinaire des locuteurs de substrats (langues autochtones) dans les contextes post-coloniaux.

26. Une jolie illustration que ce propos de Pourawa, poète kanak, commentant les vingt-huit langues kanak existant dans l'archipel calédonien : En Kanaky-Nouvelle-Calédonie le français est la $29^{e}$ langue kanak (FaceBook, 09-2014). Notons que cette langue française « langue 2 » qui devient parfois « langue 1 » est, chez de nombreux locuteurs océaniens, qui relèvent souvent des classes défavorisées, une « variété populaire de français » : c'est le français kaya décrit par Barnèche (2005), situé au pôle opposé par rapport aux « français les plus standardisés » (voir le continuum au point suivant). 
(voir le bilan historique supra), un processus inédit de décolonisation progressive est expérimenté, grâce aux Accords de Matignon (1988) et de Nouméa (1998), autour du projet de destin commun ${ }^{27}$. Ce projet d'émancipation politique, même s'il s'oriente vers une indépendance, se noue et se nouera autour du français car $c^{\prime}$ est la seule langue que la quasi totalité des habitants maîtrise à l'oral et à l'écrit.

\subsection{Continuum sociolinguistique actuel}

Le français en Nouvelle-Calédonie, comme ailleurs, y compris dans l'Hexagone, ne correspond pas au seul français standard ${ }^{28}$ mais s'inscrit dans un continuum :

- à un pôle, se situe le français standard ou les français très standardisés ${ }^{29}$ servant plutôt les fonctions réputées hautes ${ }^{30}$ en situation formelle : ce sont les « acrolectes » en usage, par exemple, en situation surveillée chez certains Français de France fraîchement immigrés ou diffusés sur France Inter, qui émet en direct depuis les années 2000 (aux cotés des radios locales), et dont un certain nombre d'émissions usent d'une langue qui tend vers la norme exogène ; ce sont également les « acrolectes » calédoniens, formes soutenues du français local, tendant vers la norme (endogène-exogène), par exemple en usage en situation surveillée chez les locuteurs de la bourgeoisie nouméenne cultivée. Les énoncés 1 infra peuvent être des illustrations de ces acrolectes locaux ${ }^{31}$.

- à l'autre pôle, se trouvent les usages à fonctions réputées basses des situations informelles comprenant le tayo ${ }^{32}$, créole à base lexicale française, « basilecte » local minoritaire - quelques milliers de locuteurs - (énoncés 2), ainsi que toutes les formes les plus distantes de la norme : les français populaires dans leurs formes locales communes (énoncés 3) ou dans des formes locales

27. «Le passé a été le temps de la colonisation, le présent est le temps du partage, par le rééquilibrage. L'avenir doit être le temps de l'identité, dans un destin commun. » (« Préambule » de l'Accord de Nouméa du 5 mai 1998, https://www.legifrance.gouv.fr/affichTexte.do?cidTexte=JORFTEXT000000555817).

28. Est-il utile de rappeler le très grand nombre de termes sujets à débats en sociolinguistique comme ailleurs. C'est bien sûr le cas de français standard, norme, français régional, variété régionale, régionalisme, variété populaire, variété familière, variété non standard, variété, registre, usage, etc. Nous ne soulignerons pas ces difficultés terminologiques à chaque occurrence de tels termes ; en revanche, nous disons ici la gêne que nous ressentons à leur usage.

29. La norme linguistique standard peut-elle être « atteinte » par les locuteurs réels dans les pratiques réelles en particulier non préméditées ? On peut considérer que la notion de norme est un artifice commode pour la description des langues, mais non une réalité homogène observable dans les corpus.

30. Nous utilisons ces termes et cette schématisation par commodité, tout en sachant que tout terme peut être controversé (voir la note 31), surtout lorsque ces termes comportent des adjectifs connotés comme « haut », « supérieur »v $v$ «bas », « inférieur ».

31. Les énoncés 1 à 13 sont des illustrations destinées à rendre un peu concrète l'image du français calédonien. Précisons que ce sont surtout des marques lexicales qui les distinguent de la norme de référence, le lexique étant, en cet état de la recherche, le domaine le mieux connu (voir Pauleau 2016, ce volume).

32. Parfois appelé autrefois « patois de Saint Louis », lieu d'où ses locuteurs sont souvent originaires. Voir Ehrhart (2016, ce volume). 
plus groupales comme le «français populaire des jeunes » ou français kaya ${ }^{33}$ (énoncés 4) et, plus rarement, les français populaires dans leurs formes hexagonales, pour peu que certains locuteurs immigrés de France les parlent.

- la partie médiane du continuum est pour sa part constituée des formes plus ou moins « déviantes » produites plutôt dans des situations semi-formelles : « mésolectes hauts » des locuteurs surveillant relativement leur langue, mais laissant place à des incursions d'expressions plus ou moins familières (particularismes calédoniens notamment ; énoncés 5) ; « mésolectes bas » des locuteurs moins contraints par la norme et usant, par exemple, de mots vulgaires (énoncé 6).

Il faut bien sûr souligner l'existence, dans ce paysage, des multiples usages intermédiaires qui, par définition, figurent dans une représentation nommée continuum. En relèvent aussi les formes de français produites par les interférences entre langues en contact. Les "français kanak » en sont des exemples, dans leurs formes « basses » comme dans leurs formes " hautes ». Il peut s'agir, par exemple, des français populaires endogènes marqués par un lexique " ethnolectal » kanak (formes « basses » ; énoncé 7) ou des français acrolectaux kanak, proches de la norme endogène par le lexique et la syntaxe mais marqués par un fort « accent » kanak (formes " hautes » ; énoncé 8). Les « français polynésiens » ( « français tahitien » (énoncé 9) ou "français wallisien » (énoncé 10)) ou le «français indonésien » sont d'autres formes, « hautes » ou " basses » (énoncé 11), que peut prendre le français calédonien. Précisons que le " français européen », forme ethnolectale européenne du français calédonien, existe aussi, avec des marques lexicales (énoncé 12) et un « accent » particulier (caractérisé notamment par une forte nasalisation). Les énoncés (11)-(13) ou (5b) illustrent bien l'hétérogénéité des pratiques qui souvent mélangent ce que l'on appelle les « variétés », confirmant encore que celles-ci, 28 , sont très perméables ${ }^{34}$.

(1) a. La mise en place [...] d'institutions novatrices comme le sénat coutumier [institution propre au gouvernement de la Nouvelle-Calédonie] a permis d'ouvrir l'espace institutionnel au système traditionnel. (énoncé écrit, auteur calédonien européen, Viratelle in Lafargue, 2012 : 3)

b. Nos langues dansent la danse de la terre... [...] Arhâ, Ajië [noms de langues kanak] [...] Frappez le tronc d'arbre creux Pour cadencer la danse sacrée [...].

\section{Voir Barnèche (2005).}

34. En complément de la note 30, précisons que nous employons le terme variété par commodité mais avec de nombreuses réserves théoriques et pratiques qui toutes ne peuvent être explicitées ici. Notamment, du point de vue théorique, on ne peut se résoudre à considérer les diverses formes de langue (diatopiques ou autres) comme des formes homogènes aux contours rigides ; or, le terme variété peut impliquer une rigidité des contours. Sur le plan pratique, le recoupement des dimensions diatopique, diastratique et diaphasique est tel, qu'il est difficile de parler de « variété » de langue sans prendre la précaution de dire qu'il ne s'agit pas d'un ensemble de traits exclusivement observables dans telle « variété ». La fréquence plus forte de tel trait dans telle « variété » est la seule observation raisonnable que l'on puisse faire. Sans de telles précautions, les commentaires du type Oui mais on dit ça aussi chez moi! ou Oui mais on dit ça aussi en France fusent en permanence. Car tel « français régional » comporte des traits communs à tel autre « français régional » ou à tel « français populaire » ou à tel « jargon professionnel », etc. 
(énoncé écrit, poète kanak, Welepane in Bogliolo, 1994 : 249 ; Pauleau, $2007: 41)$

(2) a. Sou améné léa ['1"] da paltivié, ka sou coné ké la po ['peau'] ou tlé ['tous les'] porotcha ['blanc, zoreille'] lé pa kom sa [se réfère à la peau] ou nou. (traduction de l'énoncé (3a), énoncé en tayo, écrit d'enquête, locuteur, 2016)

b. cé pa ta nou loto la ['cette voiture elle est pas à nous'] (énoncé en tayo, écrit d'enquête, locuteur, 2016)

c. nan pa pièce ou nan pa l'arjan (traduction de l'énoncé (3d), énoncé en tayo, écrit d'enquête, locuteur, 2016)

(3) a. Vous l'avez amené dans les palétuviers! [où on se fait piquer par les moustiques] Vous savez très bien qu'une peau de zoreille c'est pas comme les nous autes ! ['une peau de Métropolitain c'est pas comme notre peau']. (écrit de B.D., 2002, Teg cité par BDLP-Nouvelle-Calédonie)

b. on a été faire un coup de pêche avec mon con ['on a été faire une partie de pêche avec lui' - connotation affectueuse : 'lui, mon ami']. (oral spontané, locuteur européen, Nouméa, 1990 ; Pauleau, 2007 : 79)

c. on connaît pas ['on sait pas'] faire des belles phrases (oral d'enquête, locutrice kanak des quartiers défavorisés, Nouméa ; Barnèche, 2005 : 221)

d. yan sous ['j'ai pas de sous' ou 'y a pas de sous'] (écrit d'enquête, locuteur, 2005, se prononce [jansu] ; BDLP-Nouvelle-Calédonie)

(4) a. pour travailler dans les bureaux faut bien parler phrasé quoi ['faut bien parler quoi'] / sinon si on phrase pas c'est chômeur ['si on parle pas bien, on sera chômeur'] (rires) [...] on n'a pas les mêmes styles d'habits quoi / ceux qu'ont grandi ici c'est plutôt kaya ['c'est plutôt cool, décontracté'] (oral d'enquête, locutrice, jeune Kanak des quartiers défavorisés, Nouméa ; Barnèche, 2005 : 228, 233)

b. ça veut faire la fille blanche là [...] elle veut faire machin-qu'est-bête-là ['elle se la joue alors qu'elle est nulle'] (oral d'enquête, locutrice, jeune Kanak des quartiers défavorisés, Nouméa ; Barnèche, 2005 : 231)

(5) a. je cherche des personnes qui voudraient faire débrousser leur terrain ['faire nettoyer leur terrain'] (oral radio, locuteur européen, Nouméa, 2005, petites annonces radiodiffusées ; Pauleau, $2007: 88$ )

b. un Mélanésien qui ne sait pas parler sa langue bè c'est grave ça:// parce que qu'estce qu'il est? c'est un Mélanésien il a la peau noire [...] c'est c'est malheureux pour lui ['c'est dommage pour lui'] (oral d'enquête, locutrice kanak des quartiers défavorisés, Nouméa ; Barnèche, 2005 : 143)

(6) culotte / les flics ils ont coaltaré tout le monde ['putain les flics ils ont aligné tout le monde'] (oral spontané, locuteur européen, Nouméa, sans date)

(7) t'as pas de canon ['t'as pas d'herbe?'] mon frère? (oral d'enquête, locuteur jeune kanak, Nouméa, 2005)

notre G.I.E. a été conçu de cette façon [...] nous allons nous organiser pour atteindre cet objectif [...] nous travaillons dans la transparence (oral télévisé, locuteur, homme politique kanak, P. Neaoutyine, Nouméa, 2015 ; prononciation fortement marquée par les interférences entre langues kanak et français) 
Ahii ! Je savais pas que t'étais zozo ! [‘Ohlala ! Je savais que t’étais métropolitain !'] (écrit de B.D., attribué à un locuteur tahitien, 2004 ; l'interjection Ahii ! est surtout en usage chez les Tahitiens ; Gielbé cité par Pauleau, 2007 : 35)

(10) Lui c'est fort ['il est fort'] (écrit de presse affichant l'oral populaire wallisien, titre d'article sur un coach sportif nouméen d'origine wallisienne, Nouméa, 2013)

(11) Dans ce pays toujours aussi divisé, où la mer est jamais fini casser [sic] ['où la mer est éternelle'] (oral chanté, locuteurs jeunes, chanson publiée par Sailofa sur youtube, 2012 ; Jamais fini casser - titre de la chanson - est une expression emblématique du parler populaire indonésien en Nouvelle-Calédonie ; BDLP-Nouvelle-Calédonie)

(12) C'est pas là qu'il faut lancer l'épervier mon fils [terme d'adresse amical] [...] C'est à l'embouchure de la rivière [...] Mon pauvre diable ! [terme d'adresse amical] (énoncés écrits, auteur calédonien, 2009 ; Kurtovitch cité par BDLP-NouvelleCalédonie)

Les «Zors» fin valabs ou quoi? ['les Métropolitains: Super, hein ?'] Radiographie d'une minorité silencieuse - Qui sont-ils ? - Quels poids pèsentils ? - Qui les représente? (écrit de presse, chapeau d'un article affichant le français calédonien commun pour susciter la connivence, puis poursuivant en français standard, Nouméa, 2015)

Ce que l'on appelle français calédonien comprend l'ensemble de toutes les formes endogènes de la langue française du continuum, y compris les formes ethnolectales. Ces dernières sont perçues avant tout comme «locales » (tel mot de tel groupe pouvant être employé par un autre groupe), suscitant la connivence car constituant « notre langue » vs « la langue des Métropolitains » ${ }^{35}$. Les traits communs à tous les groupes ethnolinguistiques (traits du lexique comme de la prononciation) constituent la part majoritaire, le fonds commun du français calédonien.

Du point de vue des représentations, ajoutons que l'ambivalence règne aux deux pôles du continuum : le pôle supérieur de la norme exogène jouit d'un certain prestige chez les locuteurs calédoniens, mais est également déprécié car associé à la culture zoreille (i.e. 'métropolitaine' dans son acception péjorative) et à une certaine prétention stéréotypique du locuteur métropolitain immigré ; à l'inverse, la norme populaire ou familière endogène jouit d'un prestige de

\footnotetext{
35. Par exemple, dans (10), ce titre de presse affiche un particularisme du français populaire wallisien, l'article brossant le portrait sympathique d'un sportif d'origine wallisienne : la stratégie pour attirer l'œil du lecteur n'est autre que d'afficher ce particularisme wallisien qui suscite un sentiment de connivence linguistique et identitaire calédonien (parler en français wallisien, c'est avant tout parler un français qui se distingue du français de France et manifester un trait de l'identité locale). D’une manière générale, et souvent de façon plaisante, on aime citer les ethnolectes en français calédonien. L'expression de français indonésien en (11), par exemple, est également employée régulièrement pour plaisanter quand on veut faire de l'humour local (à prononcer avec l'accent évidemment !).
} 
connivence linguistique (voir l'énoncé 13), mais est associée à un sentiment d'insécurité linguistique et d'illégitimité face au français standardisé.

En somme, le(s) français parlé(s) en Nouvelle-Calédonie présente(nt) a priori les mêmes propriétés générales que les autres cas de figure observables ailleurs : on ne peut donc par là justifier le manque d'intérêt des chercheurs à l'égard de cette partie précise de la francophonie. Peut-être peut-on esquisser alors une autre hypothèse, d'ordre culturel, celle du peu de visibilité des productions littéraires locales. «Les lettres calédoniennes pourtant, si elles sont peu visibles sur la scène nationale ou internationale, témoignent d'une belle vitalité », affirme A. Bihan (2012 : 2). Cette vitalité en outre manifeste peu l'identité linguistique franco-calédonienne : la littérature calédonienne ne commence à faire usage du français calédonien que depuis une quinzaine d'années, la littérature plus ancienne, notamment celle que l'on trouve depuis les années 70 dans les manuels scolaires locaux, essentiellement à travers deux auteurs, J. Mariotti et G. Baudoux, est écrite dans un français très standardisé.

Ce qui confirme en tous cas que c'est bien le français, en Nouvelle-Calédonie qui n'est pas jugé digne d'intérêt, c'est que les autres langues parlées dans l'archipel, notamment les langues kanak et le créole tayo, ont fait l'objet d'un certain nombre de travaux depuis longtemps au contraire du français calédonien ${ }^{36}$.

\section{THÉMATIQUE DU NUMÉRO}

Si les travaux linguistiques sur le français de Nouvelle-Calédonie sont encore trop peu nombreux, le retard dans les études consacrées au français de NouvelleCalédonie commence à se combler, et nous disposons aujourd'hui de suffisamment de matériau pour soutenir l'hypothèse qu'il s'agit bien d'un idiome à la fois spécifique relativement aux autres usages francophones et comparable à ceux de même statut, étant donné le continuum évoqué supra. Il nous est donc apparu nécessaire et désormais possible de faire connaître à la communauté des linguistes les recherches consacrées au français calédonien, c'est le but de cet ouvrage.

Nous présenterons des éléments de description de cette langue française du bout du monde :

- d'une part, en faisant le point sur l'état actuel de la recherche menée sur le terrain, ce que font les deux premiers articles (Christine Pauleau puis Sabine Ehrhart) qui évoquent les recherches sur les « idiomes » produits par le laboratoire linguistique calédonien (français calédonien et créole tayo) ;

36. Voir Pauleau (2016, ce volume). 
- d'autre part, en intégrant le français calédonien au « reste du monde » ${ }^{37}$ par la confrontation avec d'autres langues et d'autres « géolectes » du français. Ce volet permet de mettre en évidence des faits nouveaux sur des plans divers (selon les articles) :

- sur le plan typologique, le français calédonien confronté à certaines langues régionales, présentes dans l'espace hexagonal (donc très éloignés géographiquement de la Nouvelle-Calédonie) et à certaines « variétés de français » hexagonales, met en évidence des convergences mais aussi des particularités sémantiques et pragmatiques du franco-calédonien (cf. la contribution de Didier Bottineau, confrontation de données calédoniennes et de données concernant le breton, le gallo-britto-roman, le bas-normand, le chti) ;

- en termes de description lexicographique des terrains extra-hexagonaux, une perspective panfrancophone permet de montrer que ce que l'on croyait être des faits diatopiques s'avère être plutôt des faits diastratiques (cf. la contribution d'André Thibault, confrontation des données d'Amérique du nord, des Antilles et de Nouvelle-Calédonie) ;

- la description de la langue française dans sa globalité est également en question, avec l'article de Gudrun Ledegen qui montre que certains faits décrits jusqu'ici comme " particuliers » (particularismes diatopiques ou diastratiques) peuvent être considérés comme des faits de langue française (confrontation des données réunionnaises, calédoniennes et hexagonales) ;

- entre la langue globale et les « variétés particulières ", d'autres cadres de description peuvent être distingués, comme le « français des îles ", regroupant plusieurs « variétés extra-hexagonales » (Inka Wissner, confrontation des données calédoniennes et antillaises).

On voit que ce numéro porte à la fois sur la langue et sur la variation par rapport à un " français de référence " ${ }^{38}$ (confronter des faits de variation permet d'atteindre la langue). Ce sont en outre des plans et arrière-plans divers qui sont abordés. La confrontation de langues diverses permet une approche typologique inédite (cf. la contribution de D. Bottineau), la perspective panfrancophone permet de confronter les éléments de langue, de comparer les particularismes de

37. Geste particulièrement signifiant étant donné le peu de cas qui est fait depuis toujours du terrain calédonien, certes lointain, mais, en revanche, peuplé d'une écrasante majorité de francophones réels contrairement à de nombreux autres espaces francophones dans le monde (voir la situation géo-linguistique supra).

38. L'ensemble des contributions de ce numéro adopte bien entendu un point de vue non normatif. Les faits « de variation » sont considérés comme tels par rapport au français dit « de référence », constitué par le « français parisien de la population cultivée », du moins si l'on veut résumer très succinctement. Si l'on veut, au contraire, donner de plus amples précisions sur le « français de référence » tel qu'il est considéré par les auteurs de ce numéro, on peut dire que la norme de la « bourgeoisie parisienne cultivée » n'est absolument pas une référence arrêtée mais seulement un point de repère, un outil d'analyse. On ne peut ignorer, dans la réflexion générale sur la variation du français, l'hétérogénéité des pratiques et des interactions (notamment, les « Parisiens de la bourgeoisie cultivée » n'usent pas toujours du « français normé »). Toutefois, ces réserves n'empêchent pas de prendre en compte l'existence de groupes à l'intérieur desquels la langue est soit plus fréquemment normée qu'ailleurs (c'est le cas de la « bourgeoisie parisienne cultivée » a fortiori en situation surveillée), soit plus fréquemment non conforme à la norme (dans les groupes sociaux et géographiques autres, a fortiori en situation relâchée). 
diverses régions francophones. L'écologie linguistique est concernée aussi par le laboratoire linguistique calédonien dont l'intérêt particulier est que les faits de contact linguistique y sont historiquement récents, ce qui permet d'étudier de manière saisissante la genèse des idiomes autochtones, le français calédonien et le créole tayo (cf. contribution de S. Ehrhart).

Les contributeurs espèrent ainsi :

- apporter une lumière nouvelle sur la description sociolinguistique non seulement des variétés régionales mais aussi des variétés non standard en général, et de tous les idiomes de contact;

- susciter une remise en question des outils de description (notamment les concepts de régionalisme, perspective différentielle, diglossie, diatopisme, diastratisme, variation stylistique, vernaculaire, véhiculaire, global, local, bioprogramme, écart lexical/syntaxique, relation interlocutive, parole, langue, etc.) ;

- poser une question fondamentale, sinon fondatrice, de la sociolinguistique de la francophonie : qu'est-ce que décrire les « français régionaux »? De quelle linguistique (linguistique du système ? linguistique du locuteur ?) cela relèvet-il implicitement? ;

- poser une question épistémologique essentielle : qu'est-ce que décrire les langues dans leur écologie selon un point de vue holistique?

\section{ANNEXES}

\section{Statut de la Nouvelle-Calédonie}

Les accords Matignon interviennent le 26 juin 1988 pour créer trois provinces semiautonomes et prévoir un référendum d'autodétermination pour 1998. Le référendum a été depuis repoussé à 2018. En l'attente, les accords de Nouméa du 5 mai 1998 engagent la transformation du statut de la Nouvelle-Calédonie. Les Accords de Nouméa se traduisent par la révision constitutionnelle du 20 juillet 1998. Le statut de la NouvelleCalédonie fait désormais l'objet du titre XIII de la Constitution. Les modalités de transfert de compétences de l'État aux institutions de Nouvelle-Calédonie, les règles $\mathrm{d}^{\prime}$ organisation et de fonctionnement de ces institutions ainsi que celles relatives à la citoyenneté, au régime électoral, à l'emploi et au statut civil coutumier sont renvoyées à une loi organique. La loi organique n99-209 du 19 mars 1999 reconnaît la spécificité de ce territoire d'outre-mer et met en place des mécanismes spécifiques pour les populations insulaires. Tout d'abord, le partage de la Nouvelle-Calédonie en trois provinces semi-autonomes introduit en 1988 est consacré. Chacune des ces provinces possède une assemblée délibérante qui lui est propre. Elles disposent également de représentants au Congrès de la Nouvelle-Calédonie. Ces mesures constitutionnelles et législatives soulèvent la question du statut juridique de la Nouvelle-Calédonie. Depuis la révision constitutionnelle du 20 juillet 1998, son statut fait l'objet d'un titre constitutionnel à part entière, le titre XIII. Ceci a deux conséquences. Premièrement, le statut des territoires d'outre-mer, jusqu'alors défini par l'article 74 de la Constitution, n'est plus unique. Deuxièmement, la Nouvelle-Calédonie échappe au statut général des collectivités locales défini par le titre XII de la Constitution. Toutefois, la révision constitutionnelle de 2003 l'intègre à la liste des collectivités d'outre-mer (article 72-3). 
En fait, la Nouvelle-Calédonie est une collectivité « sui generis ». Dans ce cadre, on emploie l'expression « collectivité d'outre-mer à statut particulier » pour la désigner. (http://www.collectivites-locales.gouv.fr/statuts-nouvelle-caledonie-et-polynesie)

\section{Carte extraite de Gay (2014 : 16) aimablement transmise par l'auteur}

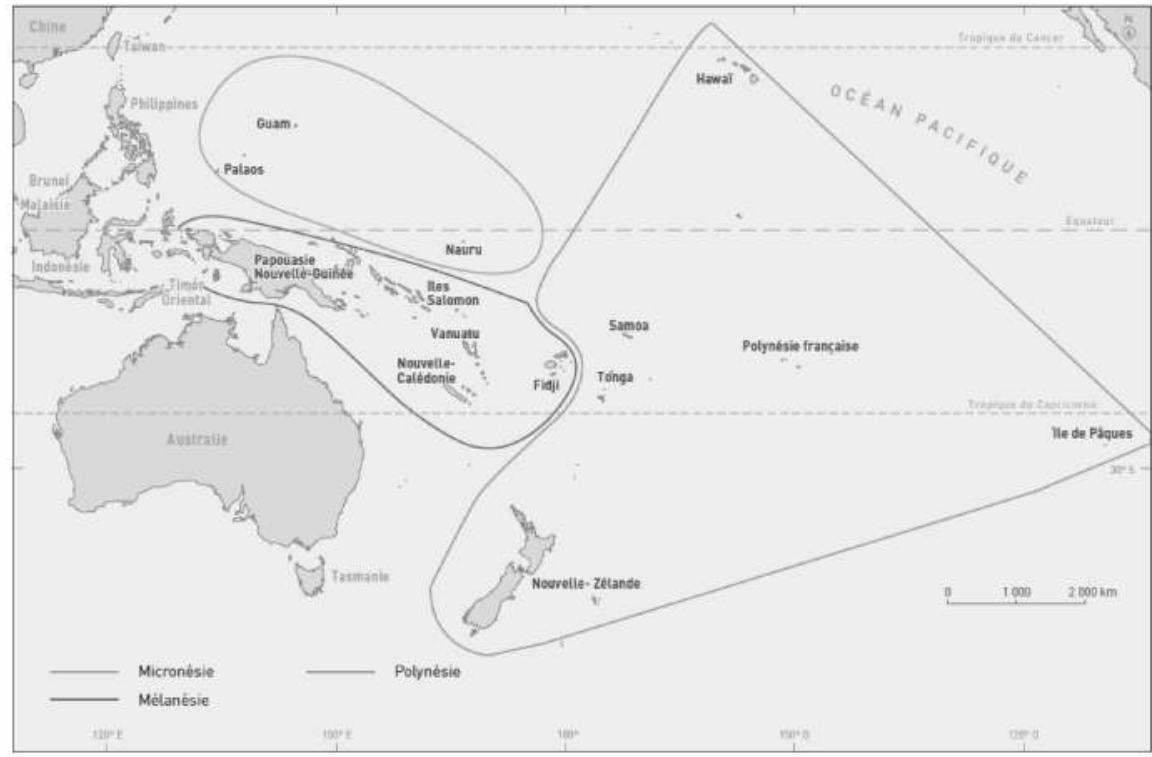

Figure 1 : Le Pacifique et ses trois aires culturelles

\section{Références}

[BDLP-NOUVELLE-CALÉDONIE] Base de données lexicographiques panfrancophones, volet " NouvelleCalédonie ", Christine Pauleau (dir.), 2006-2014. [www.bdlp.org/accueil.asp?base=NC]

[IRD] Bonvallot J., Gay J.-C. \& Habert E. (éds) (2012), Atlas de la Nouvelle-Calédonie, Marseille : Institut de Recherche pour le Développement.

BARNĖCHE S. (2005), Gens de Nouméa, gens des îles, gens d'ailleurs... Langues et identités en Nouvelle-Calédonie, Paris : L'Harmattan.

Biran A. (2012), La Nouvelle-Calédonie, ses littératures et ses livres. Bibliographie sélective et commentée, 17es Rencontres Francophones, Verson (14) : Espace Senghor \& Maison du Livre de la Nouvelle-Calédonie.

Boglıolo F. (1994), Paroles et Écritures. Anthologie de la littérature néo-calédonienne, Nouméa : Les Éditions du Cagou.

CORNE J.-C. (1979), "Le français à Tahiti ", in A. Valdman (éd.), Le Français hors de France, Paris : Honoré Champion, 631-660.

EHRHART S. (2016), "Entre français calédonien et langue kanak : quelle place pour le tayo ? Une approche écolinguistique ", Langages 203. (ce volume) 
GAY J.-C. (2014), La Nouvelle-Calédonie, un destin peu commun, Marseille : IRD Éditions.

Hollyman K. J. (1979), "Le français en Nouvelle-Calédonie ", in A. Valdman (éd.), Le Français hors de France, Paris : Honoré Champion, 621-629.

ISEE (Institut de la Statistique et des Études Économiques de la Nouvelle-Calédonie) (2011), Recensement de la population 2009, Nouméa : ISEE.

ITSEE (Institut Territorial de la Statistique et des Études Économiques) \& INSEE (Institut National de la Statistique et des Études Économiques) (1997), Recensement de la population de la Nouvelle-Calédonie : principaux tableaux 1996. Notes et documents $n^{\circ} 77$, Nouméa \& Paris : ITSEE \& INSEE.

KURTOVITCH I. (2009), Théâtre calédonien, texte non publié. (aimablement transmis par l'auteur)

Lafargue R. (2012), Le Droit coutumier en Nouvelle-Calédonie, Paris: Maison Nouvelle Calédonie.

OIF (Organisation Internationale de la Francophonie) (2005), Le Français, enjeu du xxI siècle, Paris : Nathan.

OIF (Organisation Internationale de la Francophonie) (2007), La Francophonie dans le monde 2006-2007, Paris : Nathan.

OIF (Organisation Internationale de la Francophonie) (2011), La Langue française dans le monde 2010, Paris : Nathan.

Pauleau C. (2007), Mots de Nouvelle-Calédonie, éléments de recherche sociolinguistique sur le français calédonien : inventaire lexicographique polylectal, Nouméa: Centre de Documentation Pédagogique de Nouvelle-Calédonie (2 tomes).

Pauleau C. (2016), "La description du français calédonien : état des lieux ", Langages 203. (ce volume)

QuefFÉlec A. (2000a), "Le français au Maghreb (1945-2000) ", in G. Antoine \& B. Cerquiglini (éds), Histoire de la langue française 1945-2000, Paris, Éditions du CNRS, 765-796.

QUEFFÉLEC A. (2000b), "Le français en Afrique subsaharienne francophone (1945-2000) ", in G. Antoine \& B. Cerquiglini (éds), Histoire de la langue française 1945-2000, Paris, Éditions du CNRS, 797-837. 